\title{
Complete meson-meson scattering within one loop in Chiral Perturbation Theory: Unitarization and resonances *
}

\author{
José R. Peláez and A. Gómez Nicola \\ Departamento de Física Teórica II, Universidad Complutense, 28040 Madrid, SPAIN
}

\begin{abstract}
We review our recent one-loop calculation of all the two meson scattering amplitudes within SU(3) Chiral Perturbation Theory, i.e. with pions, kaons and etas. By unitarizing these amplitudes we are able to generate dynamically the lightest resonances in meson-meson scattering. We thus obtain a remarkable description of the meson-meson scattering data right from threshold up to 1.2 $\mathrm{GeV}$, in terms of chiral parameters in good agreement with previous determinations.
\end{abstract}

Chiral Perturbation Theory (ChPT) [1] provides a remarkable description of the dynamics of pions, kaons and the eta, which are the pseudo-Goldstone bosons associated to the spontaneous $S U(3)_{L} \times S U(3)_{R}$ chiral symmetry breaking down to $S U(3)_{R+L}$. The ChPT Lagrangian contains the most general terms compatible with the symmetry breaking pattern, organized in a derivative and mass expansion (generically $p$ ), which, for the amplitudes becomes an expansion in powers of the external momenta and the masses over a scale of $\mathcal{O}(1 \mathrm{GeV})$. The loop divergences appearing at a given order can be absorbed by a finite number of constants in the Lagrangian to the same order. Thus, order by order, the theory is finite and predictive. This approach has been very successful at low energies (less than $500 \mathrm{MeV}$ ). However, it has been shown that applying a coupled channel generalization of the Inverse Amplitude Method (IAM) [2,3] one gets a very good description of meson-meson scattering up to $1.2 \mathrm{GeV}$, generating dynamically seven light resonances [ [⿴囗十: the $\rho, K^{*}, f_{0}, a_{0}$, the octet $\phi$, the $\sigma$ and the $\kappa$. The properties of the last two, and even their existence, are subject to intense debate within the hadron spectroscopy community (see these proceedings). Remarkably, the chiral amplitudes unitarized with the IAM generate poles associated to these two wide structures 沟. In principle, since this method is built from the perturbative ChPT results, it should respect the good low energy constraints. However, not all the one loop ChPT meson-meson scattering amplitudes were known. Indeed, only $\pi \pi \rightarrow \pi \pi[5$, $K \pi \rightarrow K \pi$ [5], $\eta \pi \rightarrow \eta \pi$ 朐 and $K^{+} K^{-} \rightarrow K^{+} K^{-}, K^{+} K^{-} \rightarrow K^{0} \bar{K}^{0}$ [6] were available in the literature. Therefore additional approximations had to be done [4], which spoiled partially the low energy regime and did not allow for a direct comparison with the standard ChPT parameters.

Very recently [7], we have completed the one-loop meson-meson scattering calculation. There are three new amplitudes: $K \eta \rightarrow K \eta, \eta \eta \rightarrow \eta \eta$ and $K \pi \rightarrow K \eta$, but we have recalculated the other five amplitudes unifying the notation, ensuring exact perturbative unitarity and also correcting some misprints in the literature. Next, we have applied the coupled channel IAM to these amplitudes. Our results allow for a direct comparison with the standard low-energy chiral parameters, which we find in very good agreement with previous determinations from low-energy data. The main differences with [i] are: i) we consider the full calculation of all the one-loop amplitudes in dimensional regularization, ii) we are able to describe simultaneously the low energy and the resonance regions, and iii) we pay special attention to the estimation of uncertainties.

The $\mathcal{O}\left(p^{2}\right)$ scattering amplitudes (low energy theorems) are obtained from the lowest order Lagrangian at tree level, whereas the $\mathcal{O}\left(p^{4}\right)$ calculation has the following contributions: First, the one-loop diagrams in Fig.1, which are divergent. Second, the tree level graphs with the second order Lagrangian, which depend on the chiral parameters $L_{i}$, that absorb the previous divergences through renormalization. In Table I, we list some determinations of the $L_{i}^{r}(\mu)$ renormalized in the usual $\overline{M S}-1$ scheme of ChPT, so that they depend on a scale (except $L_{3}$ and $L_{7}$ ), customarily chosen at $\mu=M_{\rho}$.

In order to compare with experiment, we use partial waves $t_{I J}$ of definite isospin $I$ and angular momentum $J$. Thus, omitting the $I, J$ subindices, the chiral expansion becomes $t \simeq t_{2}+t_{4}+\ldots$, with $t_{2}$ and $t_{4}$ of $\mathcal{O}\left(p^{2}\right)$ and $\mathcal{O}\left(p^{4}\right)$, respectively. The unitarity relation is rather simple for the partial waves $t_{i j}$, where $i, j$ denote the different available states. For example, when two states, "1" and "2", are accessible, the partial waves satisfy

$$
\operatorname{Im} T=T \Sigma T^{*} \Rightarrow \operatorname{Im} T^{-1}=-\Sigma \quad \Rightarrow \quad T=\left(\operatorname{Re} T^{-1}-i \Sigma\right)^{-1}
$$

with

$$
T=\left(\begin{array}{cc}
t_{11} & t_{12} \\
t_{12} & t_{22}
\end{array}\right) \quad, \quad \Sigma=\left(\begin{array}{cc}
\sigma_{1} & 0 \\
0 & \sigma_{2}
\end{array}\right)
$$

\footnotetext{
${ }^{*}$ To appear in the Proceedings of the IX International Conference on Hadron Spectroscopy, HADRON2001, IHEP, Protvino, Russia, August 2001
} 


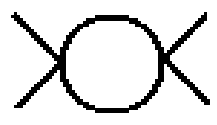

a

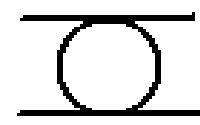

b

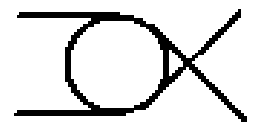

c

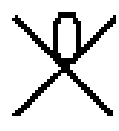

d

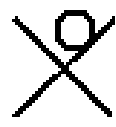

$\mathrm{e}$

FIG. 1. Generic one-loop Feynman diagrams that have to be evaluated in meson-meson scattering.

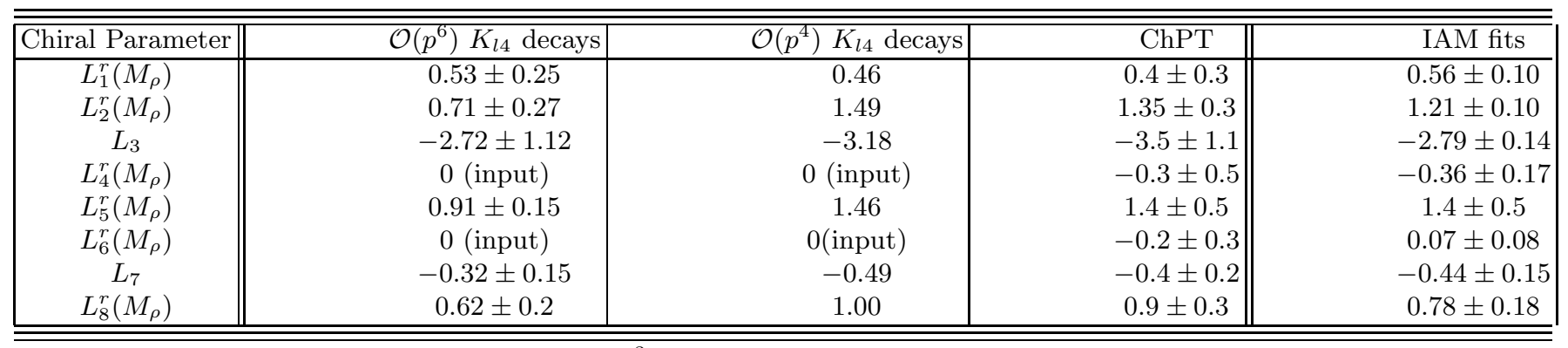

TABLE I. Several sets of chiral parameters $\left(\times 10^{3}\right)$ in the literature as well as those from an IAM fit [7], with the uncertainty due to different systematic error used on different fits.

where $\sigma_{i}=2 q_{i} / \sqrt{s}$ and $q_{i}$ is the C.M. momentum of the state $i$. It can be readily noted that we only need to know the real part of the Inverse Amplitude. The imaginary part is fixed by unitarity. Similar expressions can be obtained with $n$ accessible states. Note that, since the unitarity relations are non-linear, they will never be satisfied exactly with a perturbative expansion like that of ChPT. Still, unitarity holds perturbatively, i.e,

$$
\operatorname{Im} T_{2}=0+\mathcal{O}\left(p^{4}\right), \quad \operatorname{Im} T_{4}=T_{2} \Sigma T_{2}^{*}+\mathcal{O}\left(p^{6}\right) .
$$

A simple way to unitarize ChPT amplitudes is to use in eq.(11) the chiral expansion of $\operatorname{Re} T^{-1} \simeq T_{2}^{-1}\left(1-\left(\operatorname{Re} T_{4}\right) T_{2}^{-1}+\right.$ ...). Taking into account eq.(3), we find

$$
T \simeq T_{2}\left(T_{2}-T_{4}\right)^{-1} T_{2}
$$

which is the coupled channel IAM, which we have used to unitarize simultaneously all the one-loop ChPT meson-meson scattering amplitudes [7].

Let us remark that since we have the complete amplitudes renormalized in the $\overline{M S}-1$ scheme, we can use the IAM with previous $L_{i}^{r}$ determinations. Still we find the correct resonant behavior. Nevertheless we have carried out a fit (using MINUIT [10]) of the available data on meson-meson scattering. Since there are incompatibilities between different experiments, a 1\%,3\% and 5\% systematic error has been added, which introduces an additional source of error. We give in Table 1 the resulting chiral parameters from the fit, whose errors correspond to those of MINUIT combined with those from the systematic uncertainty. Note that they are compatible with previous determinations.

In Fig.2 we show the IAM fit (see [7] for details). The gray error bands cover the uncertainties in the $L_{i}$ due to MINUIT, and are obtained from a Monte-Carlo gaussian sampling of the parameters. Similarly, the area between the dotted lines covers the errors due to the different choice of systematic error. Note that all the resonant features are reproduced. However, since we have used the full one-loop amplitudes we are able to obtain simultaneously values for the threshold parameters (they have not been fitted) given in table 2. Note the good agreement with the experimental values.

a. Acknowledgments Work partially supported from the Spanish CICYT projects FPA2000-0956, PB98-0782 and BFM2000-1326.

[1] S. Weinberg, Physica A96, (1979) 327. J. Gasser and H. Leutwyler, Ann. Phys. 158, (1984) 142 and Nucl. Phys. B250, (1985) 465,517,539.

[2] T. N. Truong, Phys. Rev. Lett. 661, (1988) 2526 ;Phys. Rev. Lett. 67, (1991) 2260; A. Dobado, M.J.Herrero and T.N. Truong, Phys. Lett. B235, (1990) 134; 

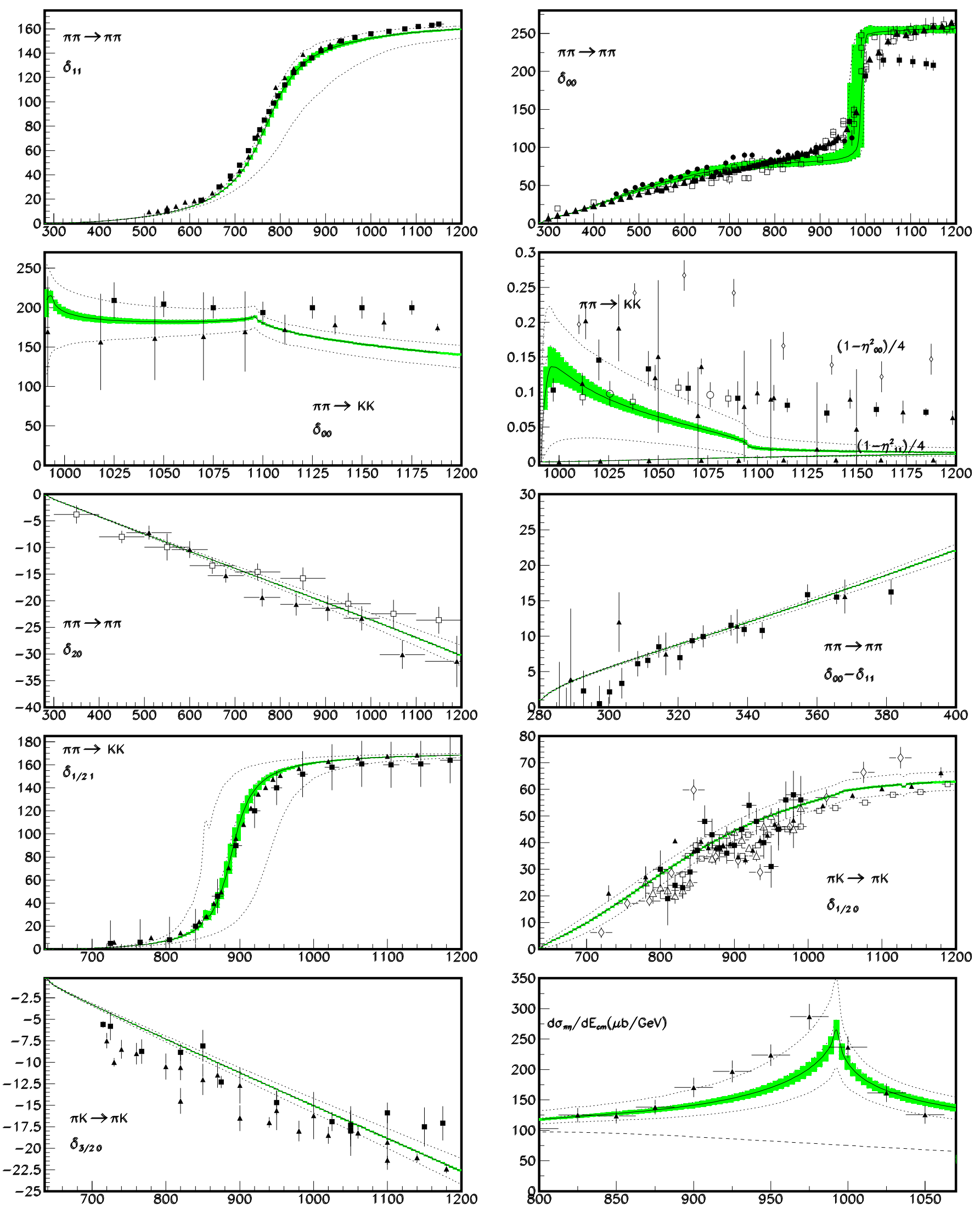

FIG. 2. Result of the coupled channel IAM fit to meson-meson scattering data (see [7] for references). The shaded area covers the uncertainty due to MINUIT errors. The area between the dotted lines corresponds to the uncertainty in the $L_{i}^{r}$ due to the use of different systematic errors on the fits. The dashed line in the last plot is the continuous background underneath the resonant contribution. 


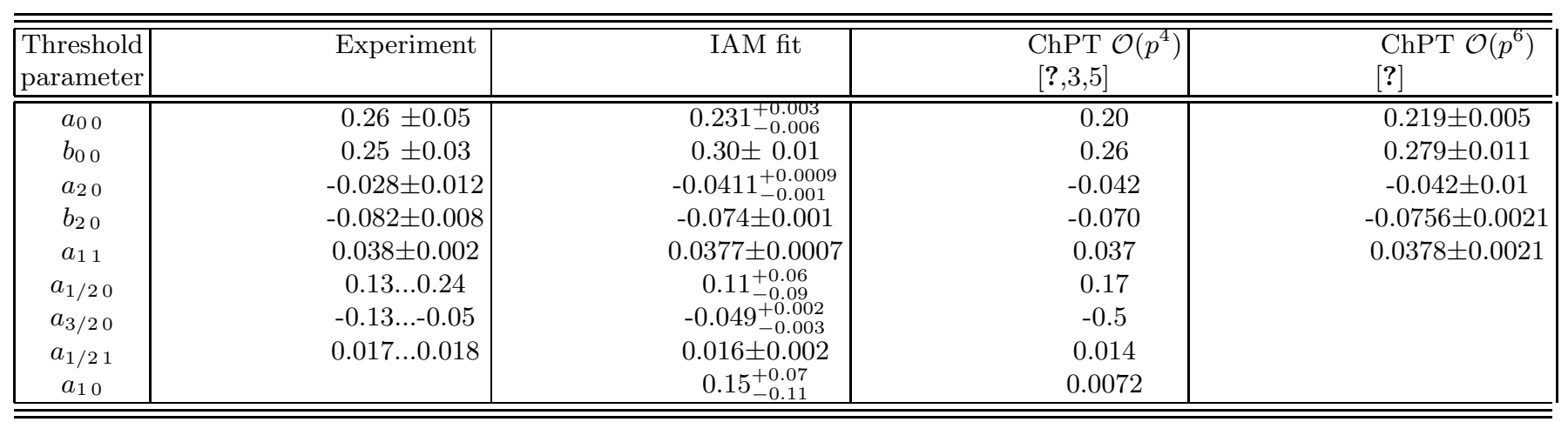

TABLE II. Meson-meson scattering lengths $a_{I J}$ and slope parameters $b_{I J}$. For experimental references see [7]. Note that our one-loop IAM results are very similar to those of two-loop ChPT.

[3] A. Dobado and J.R. Peláez, Phys. Rev. D47, (1993) 4883; Phys. Rev. D56, (1997) 3057. J. Nieves, M. Pavón Valderrama and E. Ruiz Arriola, hep-ph/0109077.

[4] J. A. Oller, E. Oset and J. R. Peláez, Phys. Rev. Lett. 80, (1998) 3452; Phys. Rev. D59, (1999) 074001; Erratum-ibid. D60, (1999) 099906.

[5] V. Bernard, N. Kaiser, U.G. Meissner, Phys. Rev. D43 (1991), 2757; Nucl. Phys. B357 (1991), 129; Phys. Rev. D44 (1991), 3698; Nucl. Phys. B364 (1991), 283.

[6] F. Guerrero and J. A. Oller, Nucl. Phys. B537, (1999) 459.

[7] A. Gómez Nicola and J. R. Peláez, hep-ph/0109056.

[8] G. Amorós, J. Bijnens and P. Talavera, Nucl. Phys. B602(2001),87. Nucl.Phys.B585:293-352,2000, Erratum-ibid.B598:665$666,2001$.

[9] J. Bijnens, G. Colangelo and J. Gasser, Nucl. Phys. B427, (1994) 427.

[10] F. James, Minuit Reference Manual D506 (1994). 